RAIRO Operations Research

RAIRO Oper. Res. 38 (2004) 305-317

DOI: $10.1051 /$ ro: 2004025

\title{
ON DUAL VECTOR OPTIMIZATION AND SHADOW PRICES
}

\author{
Letizia Pellegrini ${ }^{1}$
}

Communicated by Franco Giannessi

\begin{abstract}
In this paper we present the image space analysis, based on a general separation scheme, with the aim of studying Lagrangian duality and shadow prices in Vector Optimization. Two particular kinds of separation are considered; in the linear case, each of them is applied to the study of sensitivity analysis, and it is proved that the derivatives of the perturbation function can be expressed in terms of vector Lagrange multipliers or shadow prices.
\end{abstract}

Keywords. Vector Optimization, image space, Lagrangian duality, shadow prices.

\section{INTRODUCTION}

The recent developments in the field of Vector Optimization and the interest in its applications ask for a definition of a general scheme for carrying out the analysis of vector problems as well as for finding methods of solution. Starting from the first approaches made in [3,4], such a scheme was proposed in [5]; it is based on the image space analysis and theorems of the alternative or separation theorems, and it has produced some developments in the field of Vector Variational Inequalities besides that of Vector Optimization.

The present paper aims at presenting the image space analysis for studying the Lagrangian duality in Vector Optimization, with particular attention to the linear case and the sensitivity analysis, that is, the study of the derivatives of the

Received April 25, 2003.

1 Associate Professor, Department of Economics, University of Verona, Via Giardino Giusti 2, 37129 Verona, Italy; e-mail: letizia.pellegrini@univr.it

(C) EDP Sciences 2004 
perturbation function. Such a study is interesting because it arises in applications when the input parameters defining the problem change or contain errors. In the scalar case, some results are known: for a constrained optimization problem, the sensitivity of the optimal objective function value can be expressed in terms of the Lagrange multipliers, called shadow prices. No such simpler result is available for a vector optimization problem, because, even in the linear case, the solution set is large and non-convex, and it is no longer a unique function of the parameter. Some existing results in this direction can be found in $[1,9]$.

With the aim of defining a systematic method of research, in this paper the general scheme proposed in [5] is applied to the study of shadow prices. More precisely, two different separation approaches are proposed and compared; each of them furnishes Lagrange multipliers that can applied to the study of sensitivity analysis.

The contents of this paper are as follows. This section is completed by describing the general features of image space analysis and the separation approach. In Section 2, separation with linear scalar functions is presented, and it is proved that it shrinks to a known method, the $\varepsilon$-method. In Section 3, a different kind of separation is proposed; that is, separation with a vector function. It is compared with the previous one and it is used to define a vector Lagrangian duality, including the known dual of Isermann. In Section 4, the linear case is considered and, for each of the previous approaches, the derivatives of the perturbation function are obtained in terms of Lagrange multipliers. Finally, an example compares the two approaches.

Let the positive integers $\ell, m, n$ and the cone $D \subseteq R^{\ell}$ be given. In the sequel it will be assumed that $D$ is convex, closed and pointed with apex at the origin and with int $D \neq \emptyset$, namely with nonempty interior; $\langle\cdot, \cdot\rangle$ will denote the usual scalar product. Consider the vector-valued functions $f: R^{n} \rightarrow R^{\ell}, g: R^{n} \rightarrow R^{m}$, and the subset $X \subseteq R^{n}$. We will consider the following vector minimization problem, which is called generalized Pareto problem:

$$
\min _{D \backslash\{0\}} f(x), \quad \text { subject to } \quad x \in K:=\{x \in X: g(x)=0\},
$$

where $\min _{D \backslash\{0\}}$ denotes vector minimum with respect to the cone $D \backslash\{0\}: x^{0} \in K$ is a (global) vector minimum point (for short, v.m.p.) of (1.1), iff

$$
f\left(x^{0}\right) \nsupseteq D \backslash\{0\} f(x), \quad \forall x \in K,
$$

where the inequality means $f\left(x^{0}\right)-f(x) \notin D \backslash\{0\}$. At $D=R_{+}^{\ell}$ (1.1) becomes the classic Pareto vector problem.

It is trivial to note that $x^{0}$ is a v.m.p. of $(1.1)$, i.e. (1.2) is fulfilled, iff the system (in the unknown $x$ )

$$
f\left(x^{0}\right)-f(x) \geq_{D \backslash\{0\}} 0, \quad g(x)=0, \quad x \in X
$$


is impossible. Consider the sets:

$$
\begin{aligned}
& \mathcal{H}:=\left\{(u, v) \in R^{\ell} \times R^{m}: u \geq_{D \backslash\{0\}} 0, v=0\right\}, \\
& \mathcal{K}:=\left\{(u, v) \in R^{\ell} \times R^{m}: u=f\left(x^{0}\right)-f(x), v=g(x), x \in X\right\} .
\end{aligned}
$$

$\mathcal{H}$ and $\mathcal{K}$ are subsets of $R^{\ell} \times R^{m}$, that is called image space; $\mathcal{K}$ is called the image of problem (1.1). Now, observe that system (1.3) is impossible iff

$$
\mathcal{H} \cap \mathcal{K}=\emptyset \text {. }
$$

Hence, $x^{0}$ is a v.m.p. of (1.1) iff (1.4) holds. In general, to prove (1.4) directly may be a very difficult task. The separation approach in the image space consists in proving (1.4) indirectly by obtaining the existence of a function such that two of its disjoint level sets contain $\mathcal{H}$ and $\mathcal{K}$ respectively.

\section{Separation By SeVERAL Linear FunCtions}

The separation approach described in Section 1 can be carried out by adopting any kind of separation function, namely a linear or nonlinear function, a scalar or a vector function having any number of components. In this section, we will propose the separation with $\ell$ linear scalar functions.

Observe that system (1.3) can be equivalently split into the $\ell$ systems (in the unknown $x)$ :

$$
f_{k}\left(x^{0}\right)-f_{k}(x) \neq 0, f\left(x^{0}\right)-f(x) \in D, g(x)=0, x \in X
$$

$k \in I:=\{1, \ldots, \ell\}$.

If $D=R_{+}^{\ell}$, then the $\ell$ systems (2.1) are equivalent to

$$
f_{k}\left(x^{0}\right)-f_{k}(x)>0, f\left(x^{0}\right)-f(x) \in R_{+}^{\ell}, g(x)=0, x \in X
$$

$k \in I$, or to

$k \in I$.

$$
S_{k}\left(x^{0}\right): \quad\left\{\begin{array}{l}
f_{k}\left(x^{0}\right)>f_{k}(x) \\
f_{i}(x) \leq f_{i}\left(x^{0}\right), i \in I \backslash\{k\} \\
g(x)=0, x \in X .
\end{array}\right.
$$

Obviously (1.2) is satisfied, i.e. $x^{0}$ is a v.m.p. of (1.1), iff all the $\ell$ systems $S_{k}\left(x^{0}\right)$ are impossible. Moreover, for every $k \in I$, the impossibility of system $S_{k}\left(x^{0}\right)$ is a necessary and sufficient condition for $x^{0}$ to be a minimum point of the following scalar problem:

$$
P_{k}\left(x^{0}\right): \min f_{k}(x) \text { subject to } f_{i}(x) \leq f_{i}\left(x^{0}\right), i \in I \backslash\{k\}, g(x)=0, x \in X .
$$

On the above results, obtained by separation arguments, is based the well-known and well-studied $\varepsilon$-method (see, for example [2]), even if it has been obtained by a direct proof. More precisely, we have the following theorem. 
Theorem 2.1 (see Th. 4.3 of [2]). The solution $x^{0}$ is a v.m.p. of (1.1) iff there exist an $\varepsilon^{0} \in R^{\ell}$ such that $x^{0}$ is an optimal solution of

$$
\min f_{k}(x) \text { subject to } f_{i}(x) \leq \varepsilon_{i}^{0}, i \in I \backslash\{k\}, g(x)=0, x \in X,
$$

for all $k \in I$.

Theorem 2.1 shows that with appropriate choices of $\varepsilon$, all v.m.p. of (1.1) can be found. However, the proof of Theorem 2.1 shows that these $\varepsilon_{i}$ values are equal to $f_{i}\left(x^{0}\right)$, i.e. to the actual objective values of the v.m.p., one would like to find. Hence a confirmation or check of optimality is obtained rather than the discovery of solutions. In general, this is a deficiency of the method; nevertheless, in the study of sensitivity analysis that will be performed in Section 4, this is not a lack, because we will assume to begin with $x^{0}$, that is an optimal solution of (1.1).

Now, if we consider each of the $\ell$ scalar problems $P_{k}\left(x^{0}\right), k \in I$, we can apply the separation scheme adopted for scalar optimization [4]. Consider the sets:

$$
\mathcal{H}_{k}:=\left\{(u, v) \in R^{\ell} \times R^{m}: u_{k}>0, u_{i} \geq 0, i \in I \backslash\{k\} ; v=0\right\}, k \in I,
$$

and observe that systems $S_{k}\left(x^{0}\right)$ are all impossible iff

$$
\mathcal{H}_{k} \cap \mathcal{K}=\emptyset, k \in I
$$

For every $k \in I$, introduce the function $r_{k}: R^{\ell} \times R^{m} \rightarrow R$ given by:

$$
r_{k}\left(u, v ; \theta^{k}, \mu^{k}\right)=u_{k}+\sum_{i \in I \backslash\{k\}} \theta_{i}^{k} u_{i}+\left\langle\mu^{k}, v\right\rangle,
$$

where $\theta^{k} \in R_{+}^{\ell-1}, \mu^{k} \in R^{m}$ are parameters. It can be proved the following optimality condition (see $[4,5])$.

Theorem 2.2. Let $x^{0} \in K$. Assume that, $\forall k \in I$, there exist $\theta^{k} \in R_{+}^{\ell-1}$ and $\mu^{k} \in R^{m}$ such that

$$
f_{k}\left(x^{0}\right)-f_{k}(x)+\sum_{i \in I \backslash\{k\}} \theta_{i}^{k}\left(f_{i}\left(x^{0}\right)-f_{i}(x)\right)+\left\langle\mu^{k}, g(x)\right\rangle \leq 0, \forall x \in X,
$$

for each $k \in I$. Then $x^{0}$ is a v.m.p. of (1.1) with $C=R_{+}^{\ell}$.

\section{VECTOR SEPARATION AND VECTOR DUALITY}

In this section, we will propose a vector separation function for obtaining an optimality condition to be compared with that of Theorem 2.2. With this aim, let us set $U:=D \backslash\{0\}$ and define the vector polar of $U$ with respect to $D \backslash\{0\}$ :

$$
U_{D \backslash\{0\}}^{*}:=\left\{\Gamma \in R^{\ell \times \ell}: \Gamma u \geq_{D \backslash\{0\}} 0, \forall u \in U\right\} .
$$


Consider the function $w: R^{\ell} \times R^{m} \rightarrow R^{\ell}$ given by:

$$
w=w(u, v, \Gamma, \Lambda)=\Gamma u+\Lambda v, \quad \forall \Gamma \in U_{D \backslash\{0\}}^{*}, \quad \forall \Lambda \in R^{\ell \times m},
$$

where $\Gamma$ and $\Lambda$ are parameters. Indeed, (3.1) is a family of functions within which we will look for one in order to achieve the disjunction of $\mathcal{H}$ and $\mathcal{K}$. Define the "positive" level set of (3.1):

$$
W_{D \backslash\{0\}}(u, v, \Gamma, \Lambda):=\left\{(u, v) \in R^{\ell} \times R^{m}: w(u, v, \Gamma, \Lambda) \geq_{D \backslash\{0\}} 0\right\} .
$$

Proposition 3.1. If $w$ is given by (3.1), then we have:

$$
\begin{aligned}
& \mathcal{H} \subset W_{D \backslash\{0\}}(u, v, \Gamma, \Lambda), \quad \forall \Gamma \in U_{D \backslash\{0\}}^{*}, \quad \forall \Lambda \in R^{\ell \times m}, \\
& \mathcal{H}=\bigcap_{\substack{\Gamma \in U_{D}^{*} \\
\Lambda \in R^{\ell \times m}}} W_{D \backslash\{0\}}(u, v, \Gamma, \Lambda) .
\end{aligned}
$$

Proof. Condition (3.2a) follows immediately by the definition of $\mathcal{H}$ and of $W_{D \backslash\{0\}}$. Because of (3.2a), to show (3.2b) it is enough to prove that no element of the complement of $\mathcal{H}$ belongs to the right-hand side of $(3.2 \mathrm{~b})$; namely that $\forall(\tilde{u}, \tilde{v}) \notin \mathcal{H}$ $\exists \tilde{\Gamma} \in U_{D \backslash\{0\}}^{*}$ and $\exists \tilde{\Lambda} \in R^{\ell \times m}$ such that

$$
(\tilde{u}, \tilde{v}) \notin W_{D \backslash\{0\}}(u, v, \tilde{\Gamma}, \tilde{\Lambda}) .
$$

$(\tilde{u}, \tilde{v}) \notin \mathcal{H}$ implies at least one of the following cases: (i) $\tilde{u} \notin D \backslash\{0\}$ or (ii) $\tilde{v} \neq 0$. If (i) holds (3.3) is obtained with $\tilde{\Gamma}=I_{\ell}$ (the identity matrix of order $\ell$ ) and $\tilde{\Lambda}=0$ (the null matrix of order $\ell \times m)$, since we have $w(\tilde{u}, \tilde{v} ; \tilde{\Theta}, \tilde{\Lambda})=\tilde{u} \notin D \backslash\{0\}$. If (ii) holds, then $\exists i_{0} \in\{1, \ldots, m\}$ such that $\tilde{v}_{i_{0}} \neq 0$. Suppose that $\tilde{v}_{i_{0}}<0$. Set $\tilde{\Gamma}=\alpha I_{\ell}$ and

$$
\tilde{\Lambda}=\left(\begin{array}{ccccccc}
0 & \ldots & 0 & \tilde{d}_{1 i_{0}} & 0 & \ldots & 0 \\
\vdots & & \vdots & \vdots & \vdots & & \vdots \\
0 & \ldots & 0 & \tilde{d}_{\ell i_{0}} & 0 & \ldots & 0
\end{array}\right),
$$

where $\tilde{d}^{T}:=\left(\tilde{d}_{1 i_{0}}, \ldots, \tilde{d}_{\ell i_{0}}\right) \in D \backslash\{0\}$ and $\alpha>0$. We have $w(\tilde{u}, \tilde{v} ; \tilde{\Gamma}, \tilde{\Lambda})=\alpha \tilde{u}+\tilde{v}_{i_{0}} \tilde{d}$. Since $D$ is pointed and $\tilde{v}_{i_{0}}<0$, then $\tilde{v}_{i_{0}} \tilde{d} \notin D \backslash\{0\}$; moreover, since $\tilde{d} \neq 0$ then $\tilde{v}_{i_{0}} \tilde{d} \notin D$. Therefore, $\tilde{v}_{i_{0}} \tilde{d}$ belongs to the complement of $D$ which is open. If $\alpha$ is small enough we obtain $w(\tilde{u}, \tilde{v} ; \tilde{\Gamma}, \tilde{\Lambda})=\alpha \tilde{u}+\tilde{v}_{i_{0}} \tilde{d} \notin D$. If we suppose $\tilde{v}_{i_{0}}>0$, we repeat the same proof with $\hat{\Lambda}=-\tilde{\Lambda}$. In both cases (3.3) is shown.

Now, we are in a position to state an optimality condition for (1.1).

Theorem 3.1. Let $x^{0} \in K$. If there exist matrices $\Gamma \in U_{D \backslash\{0\}}^{*}$ and $\Lambda \in R^{\ell \times m}$ such that

$$
\Gamma\left[f\left(x^{0}\right)-f(x)\right]+\Lambda g(x) \nsupseteq D \backslash\{0\} 0, \quad \forall x \in X,
$$

then $x^{0}$ is a v.m.p. of (1.1). 
Proof. From Proposition 3.1 and (3.4) we have $\mathcal{H} \subset W_{D \backslash\{0\}}(u, v, \Gamma, \Lambda)$ and $^{1} \mathcal{K} \subset$ $\sim W_{D \backslash\{0\}}(u, v, \Gamma, \Lambda)$. Therefore (1.4) holds.

Remark 3.1. Observe that if condition (2.3) is fulfilled for each $k \in I$, then by putting

$$
\Gamma=\left(\begin{array}{llll}
1 & \tilde{\theta}_{2}^{1} & \ldots & \tilde{\theta}_{\ell}^{1} \\
\tilde{\theta}_{1}^{2} & 1 & \ldots & \tilde{\theta}_{\ell}^{2} \\
\vdots & \vdots & & \vdots \\
\tilde{\theta}_{1}^{\ell} & \tilde{\theta}_{2}^{\ell} & \ldots & 1
\end{array}\right) \quad \text { and } \quad \Lambda=\left(\begin{array}{l}
\tilde{\mu}^{1^{T}} \\
\tilde{\mu}^{2^{T}} \\
\vdots \\
\tilde{\mu}^{\ell^{T}}
\end{array}\right)
$$

it results

$$
\Gamma\left[f\left(x^{0}\right)-f(x)\right]+\Lambda g(x) \leq 0, \forall x \in X,
$$

and hence condition (3.4) is satisfied, provided that $D=R_{+}^{\ell}$. Another sufficient optimality condition that implies (3.4) is given by the following corollary.

Corollary 3.1. Let $x^{0} \in K$. If there exists $\Lambda \in R^{\ell \times m}$ such that

$$
I_{\ell}\left[f\left(x^{0}\right)-f(x)\right]+\Lambda g(x) \nsupseteq D \backslash\{0\} \quad 0, \forall x \in X,
$$

then $x^{0}$ is a v.m.p. of (1.1).

Proof. It is enough to observe that $I_{\ell} \in U_{D \backslash\{0\}}^{*}$.

The sufficient optimality condition of Corollary 3.1 can be exploited to obtain a general scheme for studying the vector Lagrangian duality related to problem (1.1). In [8], it has been defined the following vector dual problem of (1.1):

$$
\operatorname{Max}_{\Lambda} D \backslash\{0\} \min _{x \in X} D \backslash\{0\} L_{V}(x ; \Lambda),
$$

where $L_{V}(x ; \Lambda):=f(x)-\Lambda g(x)$ is called the vector Lagrangian function. Observe that in (3.5) we consider the maximum on $\Lambda$ of the set-valued map [10]:

$$
\Phi(\Lambda)=\min _{x \in X} D \backslash\{0\} L_{V}(x ; \Lambda), \Lambda \in R^{\ell \times m} .
$$

Denote by $\Delta_{1}:=\min _{x \in K} D \backslash\{0\} f(x)$ and $\Delta_{2}:=\operatorname{Max}_{\Lambda} D \backslash\{0\} \min _{x \in X} D \backslash\{0\} L_{V}(x ; \Lambda)$ the sets of optimal values of (1.1) and (3.5), respectively. In [8] it has been proved the following result.

Theorem 3.2. There exist $x^{0} \in K$ and $\Lambda^{0} \in R^{\ell \times m}$ such that

$$
\left[f\left(x^{0}\right)-f(x)\right]+\Lambda^{0} g(x) \nsupseteq D \backslash\{0\} 0, \forall x \in X
$$

iff $0 \in \Delta_{1}-\Delta_{2}$.

\footnotetext{
$1 \sim$ denotes complement.
} 
Theorem 3.2 establishes, in terms of vector separation, a necessary and sufficient condition for having an optimal solution of (1.1) and an optimal solution of its dual problem such that the corresponding optimal vector values are equal; i.e., the two problems have at least a common optimal value. We can interpret this result like a generalization of the duality gap equal to zero for scalar problems. Obviously, it is interesting to define classes of vector problems for which (3.6) is fulfilled. In [8] it has been proved that among these problems there are linear Pareto vector problems.

Let us consider the following positions: $D=R_{+}^{\ell} ; f(x)=C x$, with $C \in R^{\ell \times n}$; $g(x)=A x-b$, with $A \in R^{m \times n}$ and $b \in R^{n} ; X=\left\{x \in R^{m}: x_{i} \geq 0, i=1, \ldots, n\right\}$. Problem (1.1) becomes:

$$
\min _{R_{+}^{\ell} \backslash\{0\}} C x \text {, subject to } x \in K=\{x \geq 0, A x=b\}
$$

and its vector dual:

$$
\operatorname{Max}_{\Lambda} R_{+}^{\ell} \backslash\{0\} \min _{x \geq 0} R_{+}^{\ell} \backslash\{0\}[C x-\Lambda(A x-b)] .
$$

A known linear vector dual, that of Isermann [6], can be embedded in the formulation (3.8): let us substitute

$$
\min _{x \geq 0} R_{+}^{\ell} \backslash\{0\}[C x-\Lambda(A x-b)]
$$

with $\Lambda b$ when

$$
\begin{aligned}
& \Lambda \in T:=\left\{\Lambda \in R^{\ell \times m}:(\Lambda A-C) x \Varangle_{R_{+}^{\ell} \backslash\{0\}} 0, \forall x \geq 0\right\} \\
& =\left\{\Lambda \in R^{\ell \times m}: C x-\Lambda A x+\Lambda b \not_{R_{+}^{e} \backslash\{0\}} \Lambda b, \forall x \geq 0\right\},
\end{aligned}
$$

i.e., with one of the minimum vector values of the problem (3.9). Actually, the Isermann dual is:

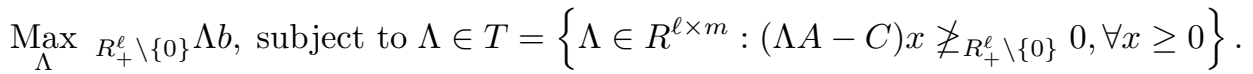

Observe that, according to Tucker theorem of the alternative (see, e.g., Th. 3, p. 29 of [7]), the feasible set $T$ of (3.10) can be equivalently defined as:

$$
T:=\left\{\Lambda \in R^{\ell \times m}: \exists \tau \in \operatorname{int} R_{+}^{\ell} \text { such that } \tau^{T}(\Lambda A-C) \leq 0\right\} .
$$

The following result holds:

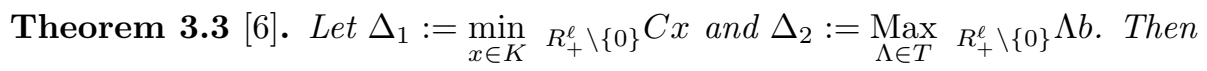

(i) $C x \not_{R_{+}^{\ell} \backslash\{0\}} \Lambda b, \forall x \in K, \forall \Lambda \in T$;

(ii) $\Delta_{1}=\Delta_{2}$. 
Moreover, a complementarity condition holds for a pair of optimal solutions of primal and dual problems.

Theorem 3.4. Let $x^{0}$ be a v.m.p. of (3.7) and $\Lambda^{0}$ an optimal solution of (3.10) such that $C x^{0}=\Lambda^{0} b$. For every $i=1, \ldots, n$ such that $x_{i}^{0}>0$ it results $\left(\Lambda^{0} A-\right.$ $C)^{i}=0$, where the apex $i$ denotes the $i$-th column of the matrix.

Proof. Obviously, $x^{0}$ and $\Lambda^{0}$ are feasible solutions of problem (3.7) and (3.10), respectively. Hence, according to the definition (3.11) of $T$, there exists $\tau \in \operatorname{int} R_{+}^{\ell}$ such that $\tau^{T}\left(\Lambda^{0} A-C\right) \leq 0$. Consider the scalar product $\left\langle\tau^{T}\left(\Lambda^{0} A-C\right), x^{0}\right\rangle$, where $x^{0} \geq 0$ is such that $A x^{0}=b$ and $C x^{0}=\Lambda^{0} b$. It turns out:

$$
\left\langle\tau^{T}\left(\Lambda^{0} A-C\right), x^{0}\right\rangle=\left\langle\tau, \Lambda^{0} A x^{0}-C x^{0}\right\rangle=\left\langle\tau^{T}, \Lambda^{0} b-C x^{0}\right\rangle=0 .
$$

Recalling that $\tau^{T}\left(\Lambda^{0} A-C\right) \leq 0$ and $x^{0} \geq 0$, the above equality to zero implies that if $x_{i}^{0}>0$ then $\tau^{T}\left(\Lambda^{0} A-C\right)^{i}=0$; since $\tau \in \operatorname{int} R_{+}^{\ell}$, the thesis follows.

\section{Sensitivity ANALYsis}

Consider the linear case, i.e. problem (3.7), and $\forall k \in I$ denote by $c^{k} \in R^{n}$ the $k$-th row of the matrix $C$. Hence the $\ell$ problems $P_{k}\left(x^{0}\right), k \in I$, become:

$L_{k}\left(x^{0}\right): \min \left\langle c^{k}, x\right\rangle$ subject to $A x=b, x \geq 0,\left\langle c^{i}, x\right\rangle \leq\left\langle c^{i}, x^{0}\right\rangle, i \in I \backslash\{k\} ;$

$k \in I$.

For each of these problems, let us consider the corresponding scalar dual problem:

$$
D_{k}\left(x^{0}\right):\left\{\begin{array}{l}
\max \left[\left\langle\mu^{k}, b\right\rangle-\sum_{i \in I \backslash\{k\}} \theta_{i}^{k}\left\langle c^{i}, x^{0}\right\rangle\right] \\
\left(\mu^{k}\right)^{T} A-\sum_{i \in I \backslash\{k\}} \theta_{i}^{k} c^{i} \leq c^{k} \\
\theta_{i}^{k} \geq 0, i \in I \backslash\{k\} .
\end{array} \quad k \in I .\right.
$$

With the aim of discussing the sensitivity in connection with the Lagrange multipliers, consider the perturbed problem:

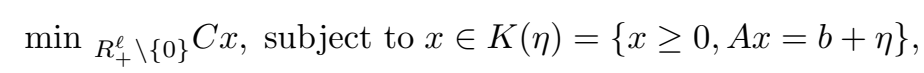

where $\eta \in R^{m}$.

In the scalar case, it is well-known that the sensitivity of the perturbation function with respect to the parameter on the right-hand side of each equality constraint is given by the corresponding Lagrange multiplier. If we want to extend this property to the vector case, we have the possibility of considering two approaches, based on the two kinds of separation exposed in the previous sections. 
In the former, we will consider Lagrange multipliers, i.e. dual variables, defined by the $\ell$ problems $D_{k}\left(x^{0}\right), k \in I$; in the latter the dual variables are represented by the matrix $\Lambda \in T$.

Let $x^{0}(\eta)$ be a v.m.p. of (4.1); therefore, by Theorem 2.1, it is also a minimum point of all the $\ell$ problems

$L_{k}(\eta): \min \left\langle c^{k}, x\right\rangle$ subject to $x \in K(\eta),\left\langle c^{i}, x\right\rangle \leq\left\langle c^{i}, x^{0}(\eta)\right\rangle, i \in I \backslash\{k\} ; \quad k \in I$.

For each $k \in I$, denote by $z_{k}^{0}(\eta)$ the optimal value of $L_{k}(\eta)$; obviously it results $z_{k}^{0}(\eta)=\left\langle c^{k}, x^{0}(\eta)\right\rangle$.

Observe that in $L_{k}(\eta)$ we have perturbed by $\eta$ only the constraints $A x=b$, while the constraints $\left\langle c^{i}, x\right\rangle \leq\left\langle c^{i}, x^{0}(\eta)\right\rangle, i \in I \backslash\{k\}$, are not originally perturbed, but they change as consequence of the movement of the optimal solution from $x^{0}$ to $x^{0}(\eta)$. For this reason, if we denote by $\left(\tilde{\theta}^{k}(\eta), \tilde{\mu}^{k}(\eta)\right)$ an optimal solution of the dual of problem $L_{k}(\eta), z_{k}^{0}(\eta)$ depends on $\tilde{\mu}^{k}(\eta), \tilde{\theta}^{k}(\eta)$ and $x^{0}(\eta)$. More precisely, we have the following result.

Theorem 4.1. Let $x^{0}(\eta)$ be a v.m.p. of (4.1) and $x_{S}^{0}(\eta)$ the sub-vector of $x^{0}(\eta)$ whose components are positive; let $\tilde{\theta}^{k}(\eta) \in R^{\ell-1}, \tilde{\mu}^{k}(\eta) \in R^{m}$ be an optimal solution of the dual of problem $L_{k}(\eta), \forall k \in I$. Then it results:

$$
\frac{\partial z_{k}^{0}(\eta)}{\partial \eta_{j}}=\tilde{\mu}_{j}^{k}(\eta)-\sum_{i \in I \backslash\{k\}} \tilde{\theta}_{i}^{k}(\eta)\left\langle c_{S}^{i}, \frac{\partial x_{S}^{0}(\eta)}{\partial \eta_{j}}\right\rangle, \forall k \in I, \forall j=1, \ldots, m
$$

Proof. $x^{0}(\eta)$ is a v.m.p. of (4.1) and hence, by Theorem 2.1, it is an optimal solution of all the $\ell$ problems $L_{k}(\eta), k \in I$. Let us consider the $k$-th of them, and denote by $x_{S}^{0}(\eta)$ the sub-vector of $x^{0}(\eta)$ whose components are positive, and by $c_{S}^{k}$ and $A_{S}$ the corresponding sub-vector and sub-matrix of $c^{k}$ and $A$, respectively. Obviously, $\forall j=1, \ldots, m$, it results:

$$
\begin{aligned}
& \frac{\partial z_{k}^{0}(\eta)}{\partial \eta_{j}}=\frac{\partial\left\langle c^{k}, x^{0}(\eta)\right\rangle}{\partial \eta_{j}}=\frac{\partial\left\langle c_{S}^{k}, x_{S}^{0}(\eta)\right\rangle}{\partial \eta_{j}}=\left\langle c_{S}^{k}, \frac{\partial x_{S}^{0}(\eta)}{\partial \eta_{j}}\right\rangle \\
&=\left\langle\tilde{\mu}^{k}(\eta)^{T} A_{S}-\sum_{i \in I \backslash\{k\}} \tilde{\theta}_{i}^{k}(\eta) c_{S}^{i}, \frac{\partial x_{S}^{0}(\eta)}{\partial \eta_{j}}\right\rangle
\end{aligned}
$$

where $\left(\tilde{\theta}^{k}(\eta), \tilde{\mu}^{k}(\eta)\right)$ is an optimal solution of the dual of $L_{k}(\eta)$ and hence the last equality holds because of the complementarity condition. 
Now, observe that $A_{S} x_{S}^{0}(\eta)-b=\eta$ and hence, by denoting with the pedex $t$ the $t$-th element of a vector or the $t$-th row of a matrix, we have, $\forall t=1, \ldots, m$ :

$$
\frac{\partial\left(A_{S} x_{S}^{0}(\eta)-b\right)_{t}}{\partial \eta_{j}}=\left(A_{S}\right)_{t} \frac{\partial x_{S}^{0}(\eta)}{\partial \eta_{j}}=\left\{\begin{array}{l}
1 \text { if } t=j \\
0 \text { if } t \neq j
\end{array}\right.
$$

Therefore, by (4.2), it results:

$$
\begin{gathered}
\frac{\partial z_{k}^{0}(\eta)}{\partial \eta_{j}}=\left\langle\tilde{\mu}^{k}(\eta)^{T}, A_{S} \frac{\partial x_{S}^{0}(\eta)}{\partial \eta_{j}}\right\rangle-\sum_{i \in I \backslash\{k\}} \tilde{\theta}_{i}^{k}(\eta)\left\langle c_{S}^{i}, \frac{\partial x_{S}^{0}(\eta)}{\partial \eta_{j}}\right\rangle \\
=\tilde{\mu}_{j}^{k}(\eta)-\sum_{i \in I \backslash\{k\}} \tilde{\theta}_{i}^{k}(\eta)\left\langle c_{S}^{i}, \frac{\partial x_{S}^{0}(\eta)}{\partial \eta_{j}}\right\rangle, \\
\forall k \in I, \forall j=1, \ldots, m .
\end{gathered}
$$

A different way of performing the sensitivity analysis is suggested by the vector dual (3.8), where the dual variable is a matrix $\Lambda \in R^{\ell \times m}$. In this scheme, if $\Lambda^{0}$ is an optimal solution of (3.8), then, $\forall k \in I$, the $k$-th row of $\Lambda^{0}$ is assumed to be a measure of the sensitivity of the the $k$-th objective $\left\langle c^{k}, x\right\rangle$ with respect to the perturbation $A x=b+\eta$ of the equality constraints. In fact, we have the following result.

Theorem 4.2. Let $x^{0}(\eta)$ be a v.m.p. of (4.1). Then there exists $\Lambda^{0}(\eta)=\left(\lambda_{k j}^{0}(\eta)\right)$, $k \in I, j=1, \ldots, m$, optimal solution of the Isermann dual of (4.1), such that

$$
\frac{\partial\left(C x^{0}(\eta)\right)_{k}}{\partial \eta_{j}}=\frac{\partial\left\langle c^{k}, x^{0}(\eta)\right\rangle}{\partial \eta_{j}}=\lambda_{k j}^{0}(\eta), \forall k \in I, \forall j=1, \ldots, m
$$

Proof. If $x^{0}(\eta)$ is a v.m.p. of $(4.1)$, then by Theorem 3.3 there exist $\Lambda^{0}(\eta)$ optimal solution of the dual of (4.1) such that $C x^{0}(\eta)=\Lambda^{0}(\eta)(b+\eta)$. Let $x_{S}^{0}(\eta)$ be the subvector of $x^{0}(\eta)$ whose components are positive, and $C_{S}$ and $A_{S}$ the corresponding sub-matrices of $C$ and $A$, respectively. By the feasibility of $x^{0}(\eta)$, it results:

$$
C x^{0}(\eta)=C_{S} x_{S}^{0}(\eta)=C_{S} x_{S}^{0}(\eta)-\Lambda^{0}(\eta)\left(A_{S} x_{S}^{0}(\eta)-b-\eta\right)
$$


Hence, if we consider the $k$-th component of $C x^{0}(\eta)$, by the above equalities, $\forall j=1, \ldots, m$, we have:

$$
\begin{aligned}
\frac{\partial\left(C x^{0}(\eta)\right)_{k}}{\partial \eta_{j}}= & \frac{\partial\left\langle c^{k}, x^{0}(\eta)\right\rangle}{\partial \eta_{j}} \\
= & \left\langle c_{S}^{k}, \frac{\partial x_{S}^{0}(\eta)}{\partial \eta_{j}}\right\rangle-\left\langle\frac{\partial\left(\Lambda^{0}(\eta)\right)_{k}}{\partial \eta_{j}}, A_{S} x_{S}^{0}(\eta)-b-\eta\right\rangle \\
& -\left\langle\left(\Lambda^{0}(\eta)\right)_{k}, A_{S} \frac{\partial x_{S}^{0}(\eta)}{\partial \eta_{j}}\right\rangle+\lambda_{k j}^{0}(\eta) \\
= & \left\langle c_{S}^{k}-\left(\Lambda^{0}(\eta)\right)_{k} A_{S}, \frac{\partial x_{S}^{0}(\eta)}{\partial \eta_{j}}\right\rangle+\lambda_{k j}^{0}(\eta) .
\end{aligned}
$$

Now, observe that $c_{S}^{k}-\left(\Lambda^{0}(\eta)\right)_{k} A_{S}=0$, because it is the $k$-th row of the sub-matrix $C_{S}-\Lambda^{0}(\eta) A_{S}$ corresponding to the positive components of $x^{0}(\eta)$; by Theorem 3.4, $C_{S}-\Lambda^{0}(\eta) A_{S}$ is the null matrix. Therefore, it turns out:

$$
\frac{\partial\left(C x^{0}(\eta)\right)_{k}}{\partial \eta_{j}}=\frac{\partial\left\langle c^{k}, x^{0}(\eta)\right\rangle}{\partial \eta_{j}}=\lambda_{k j}^{0}(\eta), \forall k \in I, \forall j=1, \ldots, m
$$

and the proof is complete.

Both results obtained by means of Theorems 4.1 and 4.2 give the partial derivatives of the perturbation function of problem (4.1) with respect to $\eta_{j}, \forall j=$ $1, \ldots, m$, i.e. the parameter on the right-hand side of each equality constraint. Nevertheless, these derivatives are expressed in a different form according to the particular kind of separation which has been chosen. This fact put in evidence the importance of the method proposed: it is a unifying scheme where different aspects of the same topic can be analysed and compared.

The following example illustrates the results of Theorems 4.1 and 4.2.

Example 4.1. Let us set $\ell=2, m=1, n=2$ and $D=R_{+}^{2}$. Consider problem (3.7) where $\left\langle c^{1}, x\right\rangle=-3 x_{1}-2 x_{2},\left\langle c^{2}, x\right\rangle=-x_{1}-4 x_{2}$ and $K=\left\{x \in R^{2}: x_{1}+2 x_{2}=\right.$ $\left.2, x_{1} \geq 0, x_{2} \geq 0\right\}$. Hence the perturbed problem (4.1) is

$$
\left\{\begin{array}{l}
\min \left(-3 x_{1}-2 x_{2},-x_{1}-4 x_{2}\right) \\
x_{1}+2 x_{2}=2+\eta \\
x_{1} \geq 0, x_{2} \geq 0
\end{array} \quad \eta \in R\right.
$$

and $x^{0}(\eta)=\left(1+\frac{\eta}{2}, \frac{1}{2}+\frac{\eta}{4}\right)$ is an optimal solution of (4.3). It results $\left\langle c^{1}, x^{0}(\eta)\right\rangle=$ $-2(2+\eta)$ and $\left\langle c^{2}, x^{0}(\eta)\right\rangle=-\frac{3}{2}(2+\eta)$; hence $\frac{\mathrm{d}\left\langle c^{1}, x^{0}(\eta)\right\rangle}{\mathrm{d} \eta}=-2$ and $\frac{\mathrm{d}\left\langle c^{2}, x^{0}(\eta)\right\rangle}{\mathrm{d} \eta}=-\frac{3}{2}$. 
$x^{0}(\eta)$ is an optimal solution also of the two problems $L_{1}(\eta)$ and $L_{2}(\eta)$ :

$$
L_{1}(\eta):\left\{\begin{array}{l}
\min \left(-3 x_{1}-2 x_{2}\right) \\
x_{1}+2 x_{2}=2+\eta \\
-x_{1}-4 x_{2} \leq-3-\frac{3}{2} \eta \\
x_{1} \geq 0, x_{2} \geq 0
\end{array} \quad \text { and } \quad L_{2}(\eta):\left\{\begin{array}{l}
\min \left(-x_{1}-4 x_{2}\right) \\
x_{1}+2 x_{2}=2+\eta \\
-3 x_{1}-2 x_{2} \leq-4-2 \eta \\
x_{1} \geq 0, x_{2} \geq 0
\end{array}\right.\right.
$$

The dual problems of $L_{1}(\eta)$ and $L_{2}(\eta)$ are:

$$
\begin{aligned}
& D_{1}(\eta):\left\{\begin{array}{l}
\max \left[\mu^{1}(2+\eta)-\theta^{1}\left(-3-\frac{3}{2} \eta\right)\right] \\
\mu^{1}+\theta^{1} \leq-3 \\
2 \mu^{1}+4 \theta^{1} \leq-2 \\
\theta^{1} \geq 0
\end{array}\right. \\
& \text { and } \quad\left\{\begin{array}{l}
\max \left[\mu^{2}(2+\eta)-\theta^{2}(-4-4 \eta)\right] \\
\mu^{2}+3 \theta^{2} \leq-1 \\
2 \mu^{2}+2 \theta^{2} \leq-4 \\
\theta^{2} \geq 0
\end{array}\right.
\end{aligned}
$$

respectively.

The optimal solution of problem $D_{1}(\eta)$ corresponding to $x^{0}(\eta)$ is $\left(\tilde{\theta}^{1}(\eta), \tilde{\mu}^{1}(\eta)\right)=$ $(2,-5)$, while that of $D_{2}(\eta)$ is $\left(\tilde{\theta}^{2}(\eta), \tilde{\mu}^{2}(\eta)\right)=\left(\frac{1}{2},-\frac{5}{2}\right)$. By Theorem 4.1, we obtain:

$$
\frac{\mathrm{d}\left\langle c^{1}, x^{0}(\eta)\right\rangle}{\mathrm{d} \eta}=\tilde{\mu}^{1}(\eta)-\tilde{\theta}^{1}(\eta)\left\langle c^{1}, \frac{\mathrm{d} x^{0}(\eta)}{\mathrm{d} \eta}\right\rangle=-5-2\left\langle(-3,-2),\left(\frac{1}{2}, \frac{1}{4}\right)\right\rangle=-2
$$

and

$\frac{\mathrm{d}\left\langle c^{2}, x^{0}(\eta)\right\rangle}{\mathrm{d} \eta}=\tilde{\mu}^{2}(\eta)-\tilde{\theta}^{2}(\eta)\left\langle c^{2}, \frac{\mathrm{d} x^{0}(\eta)}{\mathrm{d} \eta}\right\rangle=-\frac{5}{2}-\frac{1}{2}\left\langle(-1,-4),\left(\frac{1}{2}, \frac{1}{4}\right)\right\rangle=-\frac{3}{2}$

that is the same result obtained by the direct calculation of the derivative of $\left\langle c^{1}, x^{0}(\eta)\right\rangle$ and $\left\langle c^{2}, x^{0}(\eta)\right\rangle$. 
Now, consider the Isermann dual of the problem (4.3) when $\eta=0$. In this case, the dual variable is the matrix $\Lambda=\left(\begin{array}{c}\lambda_{11} \\ \lambda_{21}\end{array}\right)$ and the dual is:

$\max \left(2 \lambda_{11}, 2 \lambda_{21}\right)$ subject to

$$
\left(\begin{array}{cc}
\lambda_{11}+3 & \lambda_{11}+2 \\
\lambda_{21}+1 & \lambda_{21}+4
\end{array}\right)\left(\begin{array}{c}
w_{1} \\
w_{2}
\end{array}\right) \notin R_{+}^{2} \backslash\{0\} \forall w_{1} \geq 0, w_{2} \geq 0 .
$$

By the thesis of Theorem 4.2, we have:

$$
\left.\frac{\partial\left(C x^{0}(\eta)\right)_{k}}{\partial \eta_{j}}\right|_{\eta=0}=\lambda_{k j}^{0}, \forall k \in I, \forall j=1, \ldots, m
$$

In fact, the optimal solution corresponding to $x^{0}=\left(1, \frac{1}{2}\right)$ is $\Lambda^{0}=\left(\begin{array}{c}-2 \\ -\frac{3}{2}\end{array}\right)$ that gives the derivatives of $\left\langle c^{1}, x^{0}(\eta)\right\rangle$ and $\left\langle c^{2}, x^{0}(\eta)\right\rangle$ at $\eta=0$.

\section{REFERENCES}

[1] S. Bolintineanu and B.D. Craven, Linear multicriteria sensitivity and shadow costs. Optimization 26 (1992) 115-127.

[2] M. Ehrgott, Multicriteria Optimization. Springer, Lect. Not. Econom. Math. Syst. 491 (2000).

[3] F. Giannessi, Theorems of the alternative, quadratic programs and complementarity problems, in Variational Inequalities and Complementarity Problems, edited by R.W. Cottle et al. J. Wiley (1980) 151-186.

[4] F. Giannessi, Theorems of the alternative and optimality conditions. J. Optim. Theor. Appl. 42 (1984) 331-365.

[5] F. Giannessi, G. Mastroeni and L. Pellegrini, On the theory of vector optimization and variational inequalities. Image space analysis and separation, in Vector Variational Inequalities and Vector Equilibria. Mathematical Theories, edited by F. Giannessi. Kluwer Acad. Publ. (2000) 153-215.

[6] H. Isermann, On some relations between a dual pair of multiple objective linear programs. Z. Oper. Res. 22 (1978) 33-41.

[7] O.L. Mangasarian, Nonlinear Programming. SIAM Classics Appl. Math. 10 (1994).

[8] L. Pellegrini, On Lagrangian duality in vector optimization. Optimization. Submitted.

[9] T. Tanino, Sensitivity analysis in multiobjective optimization. J. Optim. Theor. Appl. 56 (1988) 479-499.

[10] W. Song, Duality for vector optimization of set valued functions. J. Math. Anal. Appl. 201 (1996) 212-225. 